\title{
Optical Measurement of Magnetic Anisotropy Field in Nanostructured ferromagnetic Thin Films
}

\author{
Hyun-Seok Whang, Sang-Jun Yun, Joon Moon, and Sug-Bong Choe* \\ Department of Physics and Institute of Applied Physics, Seoul National University, Seoul 151-742, Korea
}

(Received 15 January 2015, Received in final form 10 February 2015, Accepted 11 February 2015)

\begin{abstract}
The magnetic anisotropy field plays an important role in spin-orbit-torque-induced magnetization dynamics with electric current injection. Here, we propose a magnetometric technique to measure the magnetic anisotropy field in nanostructured ferromagnetic thin films. This technique utilizes a magneto-optical Kerr effect microscope equipped with two-axis electromagnets. By measuring the out-of-plane hysteresis loops and then analyzing their saturated magnetization with respect to the in-plane magnetic field, the magnetic anisotropy field is uniquely quantified within the context of the Stoner-Wohlfarth theory. The present technique can be applied to small nanostructures, enabling in-situ determination of the magnetic anisotropy field of nanodevices.
\end{abstract}

Keywords : perpendicular magnetic anisotropy field, magneto optical Kerr effect, hysteresis loop, Stoner-Wohlfarth model

\section{Introduction}

Magnetic domain-wall memory devices [1] have attracted a great deal of attention as potential candidates of emerging memory devices. Of such devices, the most common is nanostructured ferromagnetic thin films with perpendicular magnetic anisotropy (PMA) [2]. The effective field induced by the PMA - namely, the magnetic anisotropy field has been found to play an important role in magnetization dynamics driven by the spin-orbit torque (SOT) [3-6]. Therefore, precise measurement of the magnetic anisotropy field is becoming crucial nowadays to understand the SOT-induced magnetization dynamics as well as to quantify the magnitude of the SOT. To measure such magnetic anisotropy fields, torque magnetometry [7] has been the conventional technique for bulk materials, but this technique is not applicable to nanostructured devices due to their small volume and consequently weak signal. Recently, magnetometry based on the extraordinary Hall effect (EHE) has been proposed to measure the magnetic anisotropy field from ferromagnetic thin films [8]. However, this technique requires specific measurement geometry to inject the electric current into the structure,

CThe Korean Magnetics Society. All rights reserved.

*Corresponding author: Tel: $+82-2-880-9254$

Fax: +82-2-880-9254, e-mail: sugbong@snu.ac.kr and exhibits a number of artifacts that require sophisticated and time-consuming analysis techniques. Here, we propose another magnetometric technique based on optical measurement, which allows the magnetic anisotropy field to be measured from nanostructured devices without any modification of specific measurement geometry.

\section{Experimental Results and Discussions}

Figure 1 shows a schematic diagram of the measurement setup. The magnetic signal from the sample is measured by using a magneto-optic Kerr effect (MOKE) microscope with a spatial resolution better than $3 \mu \mathrm{m}$ and an objective lens with a numerical aperture $(0.45)[9,10]$. This microscope is equipped with two electromagnets to apply the in-plane and out-of-plane magnetic fields up to $600 \mathrm{mT}$ and $150 \mathrm{mT}$, respectively.

The present measurement setup was applied to ferromagnetic $\mathrm{Pd} / \mathrm{Co} / \mathrm{Pt}$ films. All the films exhibit PMA. The detailed sample structure is $5.0-\mathrm{nm} \mathrm{Ta} / 1.8-\mathrm{nm} \mathrm{Pd} / 0.3-\mathrm{nm}$ $\mathrm{Co} / 2.5-\mathrm{nm} \mathrm{Pt}$, which was prepared on an $\mathrm{Si}$ substrate with a 100 -nm-thick $\mathrm{SiO}_{2}$ layer by dc magnetron sputtering. For the measurement of the magnetic anisotropy field, an in-plane magnetic field bias $H_{\text {in }}$ was applied to the sample, and the out-of-plane magnetic hysteresis loop was then measured by sweeping the out-of-plane magnetic field $H_{\text {out }}$. Figure 2 shows the magnetic hysteresis loops mea- 


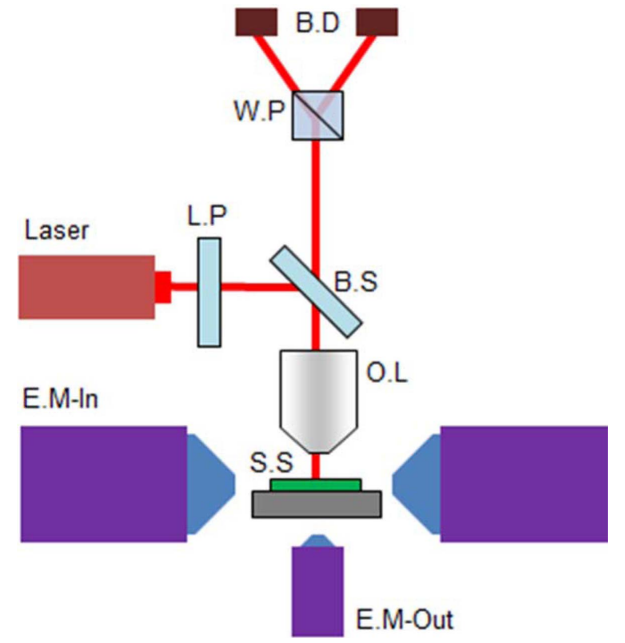

Fig. 1. (Color online) Schematic description of the experimental setup. Here, E.M-In is the in-plane electromagnet and E.MOut is the out-of-plane electromagnet. S.S refers to the sample stage, O.L denotes the objective lens, B.S refers to the beam splitter, and L.P denotes the linear polarizer. W.P refers to the Wollaston prism and B.D. denotes the balanced detector.

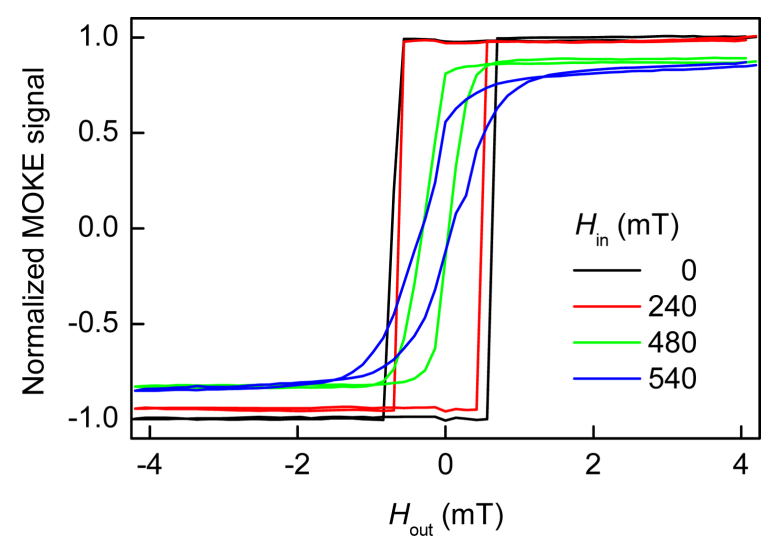

Fig. 2. (Color online) Out-of-plane magnetic hysteresis loops for various $H_{\text {in }}$ denoted inside the figure. The ordinate is the MOKE signal normalized by the saturated magnetization measured from the hysteresis loop with $H_{\text {in }}=0$.

sured under various in-plane field biases $H_{i n}$. The figure clearly shows that the shape of the hysteresis loops is sensitive to the strength of $H_{i n}$.

The variation of the hysteresis loops can be explained within the context of the Stoner-Wohlfarth theory, in which the magnetic energy $E$ is given by $E=K_{U} \sin ^{2} \theta-$ $M_{S} H \cos (\theta-\varphi)$, where $K_{U}$ is the uniaxial magnetic anisotropy constant with the out-of-plane easy axis, $M_{S}$ is the saturation magnetization, and $H$ is the strength of the external magnetic field. Here, the angle $\theta$ of the magnetization and the angle $\varphi$ of the external magnetic field are defined as the polar angles with respect to the easy axis.
The magnetic anisotropy field $H_{K}$ is then defined as $H_{K}=$ $2 K_{U} / M_{S}[11,12]$. Since the present experimental geometry gives $H_{\text {in }}=H \sin \varphi$ and $H_{\text {out }}=H \cos \varphi$, the magnetic energy can be written as

$$
E=\frac{M_{S} H_{K}}{2} \sin ^{2} \theta-M_{S} H_{\text {out }} \cos \theta-M_{S} H_{\text {in }} \sin \theta .
$$

The equilibrium angle $\theta_{e q}$ is then obtained by the energy minimization condition i.e.

$$
\begin{aligned}
\left.\frac{\partial E}{\partial \theta}\right|_{\theta_{e q}}= & M_{S} H_{K} \sin \theta_{e q} \cos \theta_{e q}+M_{S} H_{o u t} \sin \theta_{e q} \\
& -M_{S} H_{\text {in }} \cos \theta_{e q}=0 .
\end{aligned}
$$

The exact solution of Eq. (2) is fairly complicated. However, two special cases exist with simple solutions, which consequently provide two distinct measurement schemes.

The first scheme (Scheme 1) is based on the saturated $\theta_{e q}$ measured under a $H_{\text {out }}$ larger than the coercive field. For this case, one can easily obtain the solution as $\theta_{\text {eq }}=M_{S} H_{\text {in }} /\left(M_{S} H_{K}+M_{S} H_{\text {out }}\right)$ or

$$
\cos \theta_{\text {eq }} \cong 1-\frac{1}{2} \frac{H_{\text {in }}^{2}}{\left(H_{K}+H_{\text {out }}\right)^{2}},
$$

for a small $\theta_{e q}$. Note that $\cos \theta_{e q}$ can be easily measured experimentally from the normalized magnetic hysteresis loops, as seen in Fig. 2. Figure 3(a) shows the plot of $\cos \theta_{e q}$ with respect to $H_{i n}$, measured for $H_{\text {out }}=4 \mathrm{mT}$. It is clear from the figure that the measured $\cos \theta_{e q}\left(H_{i n}\right)$ follows the behavior predicted by Eq. (3). The red line shows the best fit with Eq. (3), which quantifies $H_{K}$ as $1013 \pm 50 \mathrm{mT}$.

The second scheme (Scheme 2) is proposed to analyze $H_{K}$ based on the measurement of the remnant $\cos \theta_{e q}$ with $H_{\text {out }}=0$ [13]. For this case, Eq. (2) can be simplified to $M_{S} H_{K} \sin \theta_{e q} \cos \theta_{e q}-M_{S} H_{\text {in }} \cos \theta_{e q}=0$, providing the solutions $\sin \theta_{e q}=H_{i n} / H_{K}$ and $\cos \theta_{e q}=0$. Since the latter solution is found to be unstable, the unique stable solution consequently provides the relation,

$$
\cos \theta_{e q}=\sqrt{1-\left(H_{i n} / H_{K}\right)^{2}} .
$$

However, we found that this analysis method fails to quantify $H_{K}$. The symbols in Fig. 3(b) show the measured $\cos \theta_{e q}$ with respect to $H_{i n}$. The bluedashed lines show the curves of Eq. (4) with different values of $H_{K}$ as denoted inside the plot. It is clear from the figure that none of the curves of Eq. (4) fit the experimental data. The failure is possibly caused by either the multi-domain formation or the large variation of the magnetization direction due to the irregularities in the anisotropy at the remnant states. The best fitting value for small $H_{\text {in }}(<300 \mathrm{mT})$ is similar to that obtained by Scheme 1, but the best fitting value for 


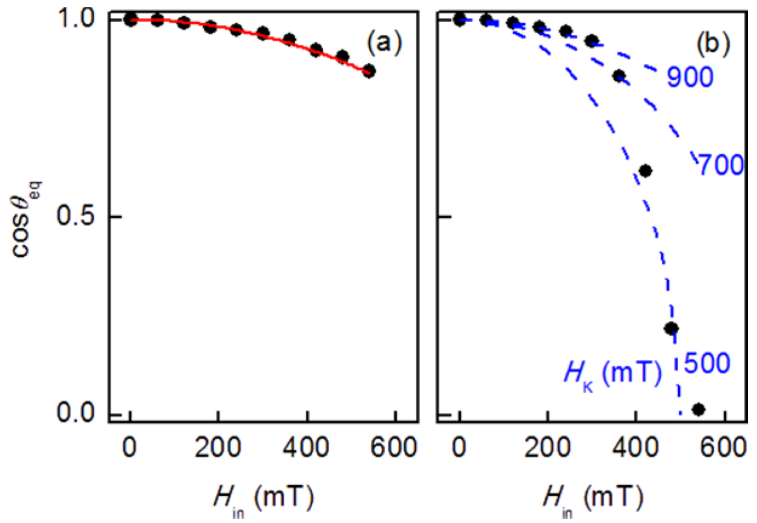

Fig. 3. (Color online) Plots of (a) the saturated $\cos \theta_{e q}$ with $H_{\text {out }}=4 \mathrm{mT}$ and (b) the remnant $\cos \theta_{\text {eq }}$ with $H_{\text {out }}=0 \mathrm{mT}$, measured with respect to various $H_{\text {in }}$. The red solid line in (a) shows the best fits with Eq. (3). The blue dashed lines in (b) show the curves of Eq. (4) with several different $H_{K}$ as denoted inside the plot.

all the data occurs when $H_{K}$ is about $500 \mathrm{mT}$, which considerably differs to that obtained by Scheme 1 .

To examine the validity of the value obtained by Scheme 1, we compare it with the value measured by the electrical method based on the EHE measurement [8]. Due to the sizeable ordinary and planar Hall effects observed in these samples, the electrical measurement is accompanied with sizeable artifacts caused by the ordinary and planar Hall effects. By measuring these artifacts from their different angular dependence between the magnetic field and the magnetization directions, the best estimation of $H_{K}$ is found to be $952 \pm 62 \mathrm{mT}$, which is accordant with the value determined by Scheme 1 within the experimental accuracy. One can therefore conclude that Scheme 1 (rather than Scheme 2) provides better accuracy in the $H_{K}$ measurement. It is also worth noting that the present optical scheme provides an easier way to analyze $H_{K}$ compared to the electrical method that requires sophisticated analysis of the artifacts, including the ordinary and planar Hall effect.

\section{Conclusions}

In conclusion, we propose an optical magnetometric technique by using a MOKE microscope equipped with two electromagnets. By measuring the out-of-plane hysteresis loops under various in-plane magnetic field biases, the magnetic anisotropy field is quantified based on the Stoner-Wohlfarth theory of the magnetization rotation. Due to the nature of the optical measurement scheme, the present technique can be applied to a local spot of nanostructured devices, enabling a versatile diagnosis on actual devices.

\section{Acknowledgement}

This work was supported by a grant from the National Research Foundation of Korea (NRF) funded by the Ministry of Science, ICT, and Future Planning of Korea (MSIP) (2012-003418).

\section{References}

[1] S. S. P. Parkin, M. Hayashi, and L. Thomas, Science 320, 190 (2008).

[2] Ioan Mihai Miron, Kevin Garello, Gilles Gaudin, PierreJean Zermatten, Marius V. Costache, Stéphane Auffret, Sébastien Bandiera, Bernard Rodmacq, Alain Schuhl, and Pietro Gambardella, Nature 476, 189 (2011).

[3] Kevin Garello, Ioan Mihai Miron, Can Onur Avci, Frank Freimuth, Yuriy Mokrousov, Stefan Blügel, Stéphane Auffret, Olivier Boulle, Gilles Gaudin, and Pietro Gambardella, Nat. Nanotech. 8, 587 (2013).

[4] A. V. Khvalkovskiy, V. Cros, D. Apalkov, V. Nikitin, M. Krounbi, K. A. Zvezdin, A. Anane, J. Grollier, and A. Fert, Phys. Rev. B 87, 020402 (2013).

[5] P. Gambardella and I. M. Miron, Philosophical Transactions of the Royal Society A: Mathematical, Physical and Engineering Sciences 369, 3175 (2011).

[6] Ioan Mihai Miron, Gilles Gaudin, Stéphane Auffret, Bernard Rodmacq, Alain Schuhl, Stefania Pizzini, Jan Vogel, and Pietro Gambardella, Nat. Mater. 9, 230 (2010).

[7] W. H. Meiklejohn and C. P. Bean, Phys. Rev. Lett. 102, 1413 (1956).

[8] Kyoung-Woong Moon, Jae-Chul Lee, Sug-Bong Choe, and Kyung-Ho Shin, Rev. Sci. Instrum. 80, 113904 (2009).

[9] Kab-Jin Kim, Jae-Chul Lee, Sung-Min Ahn, Kang-Soo Lee, Chang-Won Lee, Young Jin Cho, Sunae Seo, KyungHo Shin, Sug-Bong Choe \& Hyun-Woo Lee, Nature 458, 740 (2009).

[10] D. A. Allwood, G. Xiong, C. C. Faulkner, D. Atkinson, D. Petit, R. P. Cowburn, J. Phys. D: Appl. Phys. 36, 2175 (2003).

[11] C. Tannous and J. Gieraltowski, Eur. J. Phys. 29, 475 (2008).

[12] D. Jiles, Introduction to Magnetism and Magnetic Materials, Chapman \& Hall, London (1998) pp. 370-375.

[13] Uwe Bauer, Lide Yao, Aik Jun Tan, Parnika Agrawal, Satoru Emori, Harry L. Tuller, Sebastiaan van Dijken, and Geoffrey S. D. Beach, Nat. Mater, Published Online (2014). 